\title{
ON THE NON-PRIMITIVE SUBSTITUTION-GROUPS OF DEGREE TEN.
}

BY DR. G. A. MILLER.

A LIST of the non-primitive substitution-groups of degree ten is given in the Quarterly Journal of Mathematics, vol. 27, pp. 40-42. By comparing with this list the theorems given below it will be found that the following six groups should be deleted :

$$
\begin{aligned}
& 200_{2}=\left\{(a b c d e)_{20}(f g h i j)_{20}\right\} q \operatorname{lad}_{1}(a f b g . c h e j \cdot d i) \\
& 200^{2}=(a b c d e)_{10}(f g h i j){ }_{10}(a f b g . c h e j . d i) \\
& 200_{8}=\left(a b c d e{ }_{10}^{10}(f g h i j){ }_{10}(a j b h . c f e g . d i)\right. \\
& 100^{8}=\left\{(a b c d e)_{10}(f g h i j)_{10}\right\} \operatorname{dim}(a j b h . c f e g . d i) \\
& 50_{2}^{3}=(a b c d e)_{5}{ }_{5}(f g h i j)_{5}(a f . b h \cdot c j \cdot d g \cdot e i) \\
& 50_{3}^{2}=(a b c d e)_{5}(f g h i j)_{5}(a f \cdot b j \cdot c i \cdot d h \cdot e g)
\end{aligned}
$$

Theorem I. There are five and only five non-primitive groups of degree ten and order two hundred.

The only bases that can be used are

$$
\begin{aligned}
& 100_{4}=\left\{\begin{array}{l}
(a b c d e)_{20}(f g h i j)_{20} \\
100_{6}
\end{array}\right\}\left\{\begin{array}{c}
\text { quad }_{1} \\
10 b c d e)_{20}(f g h i j)_{20}
\end{array} \text { quad }_{2}\right. \\
& 100_{2}=(a b c d e)_{10}(f g h i j)_{10}
\end{aligned}
$$

Since all of these involve one and only one cycle of five letters and its powers in each system and $(a b c d e)_{20}(\text { f } g h i j)_{20}$ contains all the substitutions, whose degree does not exceed ten, which transform

$$
\text { (abcde) cyc (fghij) cyc }
$$

into itself, it follows that there cannot be more than four hundred substitutions which transform any one of these bases into itself and also interchange the systems. Two hundred of these are positive and two hundred negative.

The substitution

$$
a f . b g . c h . d i \cdot e j
$$

may evidently be used to generate a tail to each one of these bases,* and as these bases together contain two hundred dif-

* The conditions which such a substitution must satisfy to generate a tail containing as many substitutious as the head contains, in case the letters are divided into two equal systems, are given in the article to which we referred in the first paragraph. They are: (1) it must transform the base into itself; (2) it must interchange the systems of intransitivity; and (B) its square must be found in the base. 
ferent substitutions we must employ all the possible negative substitutions in making up the three tails for the given bases, or heads. As the groups just obtained are distinct, we have now three non-primitive groups of degree ten and order two hundred.

It should be observed that since all the four hundred possible substitutions transform ( $a b c d e$ ) cyc ( $f g h i j)$ cyc into itself, we may prove that one of these substitutions transforms any one of the given bases into itself by showing that it transforms the remaining generating substitutions into substitutions of the base. In the first and second cases we may select one substitution as the remaining generating substitution, but in the third case we cannot select less than two.

We now inquire whether the one hundred negative substitutions which are not employed in each individual case can be used as a tail in that case, and, if so, whether the group thus obtained is distinct from those already given. In the first and third cases one of these remaining negative substitutions is

$$
\begin{array}{r}
a f \cdot b g \cdot c h \cdot d i \cdot e j \times a c e b \cdot f g j h^{*}=a g \cdot b j \cdot c f \cdot d i \cdot e h \\
=f g j^{-1} \times a f \cdot b g \cdot c h \cdot d i \cdot e j \times f g j h
\end{array}
$$
that

Since $f g j h$ transforms these bases into themselves, it follows

$$
a g \cdot b j \cdot c f \cdot d i \cdot e h
$$

may be used to generate a tail to each of these heads, but that the groups thus obtained are not distinct from the three already given.

In the second case one of these one hundred remaining negative substitutions is

$$
a f . b g . c h . d i \cdot e j \times a b c d \cdot f g h i=a g c i . b h d f \cdot e j
$$

Since agci. bhdf . ej satisfies the three necessary and sufficient conditions specified in the foot-note, it may be used to generate a tail to the second head, and it remains only to show that the group thus generated is distinct from the three already found. To do this it is only necessary to show that it is distinct from the one of these three which has the same base. If it were not distinct from this it would contain a substitution of the form

$$
a f \cdot b g \cdot c h \cdot d i \cdot e j
$$

* We have employed the notation of Professor CAYLEY as given in the Quarterly Journal of Mathematics, vol. 25, p. 77, in writing (abcde) and $(a b c d e)_{10}$. To obtain the notation employed in the list to which we referred we have to transform with respect to $c d$. $h i$. 
and beginning with $a f$; for if a particular substitution of this form would not begin with af. it could be made to do so by transforming with respect to some substitution in ( $f g h i j)$ cyc. The only substitutions in our group beginning with af . are

$$
\begin{aligned}
& \text { af . bieh . cgdj } \\
& \text { af . bjeg . cidh } \\
& \text { af . bgej . chdi } \\
& \text { af . bhei. cjdg }
\end{aligned}
$$

and as these do not have the required form our group must be distinct from the three which have already been given.

We now proceed to the positive substitutions. One of these is

$$
\text { afbgchdi } . e j
$$

Its square is found only in the first base, and it is easily seen that it may be used to generate a tail to this base, thus giving rise to the fifth and last group included in our theorem. For one of the remaining one hundred positive substitutions is

$$
\begin{aligned}
& \text { afbgchdi } . e j \times a b c d . f g j h=a g d i b j e h . c f \\
& =\overline{b d e c \cdot f g i h j}^{-1} \times a f b g c h d i \cdot e j \times b d e c \cdot f g i h j
\end{aligned}
$$

Since $b d e c$. fgihj transforms the first base into itself, it follows that a group is obtained by combining agdibjeh. $c f^{\prime}$ with this base, which is not distinct from the preceding group. The two hundred positive substitutions form two conjugate tails to the first base, hence all their squares are found in this base. From this it readily follows that all these positive substitutions are of the form afbgchdi.$e j$, and that none of their squares are found in either of the other bases. This completes the proof of the theorem.

Theorem II. There are two and only two non-primitive groups of order one hundred and degree ten.

The only possible base is

$$
\left\{(a b c d e)_{10}(f g h i j)_{10}\right\} \operatorname{dim} .
$$

From what precedes it is readily seen that the only admissible substitutions for tails are the two hundred negative substitutions which have already been employed.

$$
a f \cdot b g \cdot c h \cdot d i \cdot e j
$$


can be used to generate a tail, and we thus employ fifty of the possible substitutions. By transforming this group with respect to $f g h i$ we obtain a group having the same head but a different tail, as

$$
a g \cdot b h \cdot c i \cdot d f \cdot e j
$$

is not contained in the preceding group. We have now used one hundred of the possible substitutions and obtained only one distinct group. The substitution

$$
a g c i . b h d f \cdot e j
$$

which we used in a preceding case is not included in these one hundred, and it may be used to generate a tail, which may be easily proved by the method employed under the first theorem to be distinct from the tails already found. We thus obtain a second distinct group, and if we transform this with respect to

$$
\text { fghi }
$$

we obtain a group which has the same head but a different tail from the last, since

$$
a h c f \cdot b i d g \cdot e j
$$

is not found in the three preceding tails. We have now exhausted the two hundred possible substitutions and found only two distinct groups.

Theorem III. There is one and only one non-primitive group of degree ten and order fifty.

The only base which can be used is

$$
\text { (abcde) cyc (fghij) cyc. }
$$

Since it has been proved that all the positive substitutions which interchange these cycles are of the form

$$
\text { afbgchdi . ej, }
$$

none of their squares is contained in this base and they can therefore not be used to generate a tail. Under Theorem I. we found the following negative substitutions:

$$
\begin{aligned}
& a f . b i e h . c g d j \\
& a f . b j e g \cdot c i d h \\
& \text { af. bgej . chdi } \\
& \text { af . bhei . cjdg }
\end{aligned}
$$


By transforming these with respect to

$$
\text { (fghij) cyc }
$$

we find that there are twenty different substitutions of this form and beginning with

$$
\text { aß. } \beta=f, g, h, i, j
$$

among the two hundred possible negative substitutions. By transforming these twenty with respect to

$$
\text { (abcde) cyc }
$$

we find one hundred such negative substitutions, which are all different and begin with

$$
\alpha \beta . \quad \begin{aligned}
& \alpha=a, b, c, d, e \\
& \beta=f, g, h, i, j
\end{aligned}
$$

Since the square of none of these is found in our base, there cannot be more than one hundred substitutions available to construct tails to it.

$$
a f \cdot b g \cdot c h \cdot d i \cdot e j
$$

may be used to generate one tail, and we proceed to show that the twenty-five substitutions obtained in this way may be transformed into the seventy-five other possible substitutions by means of substitutions which transform the base into itself.

If we transform the given group with respect to

$$
g j \cdot h i
$$

we obtain a new group, as $a f . b j . c i . d h . e g$ is not found in the preceding group, there being only one substitution in it beginning with af.: then we may transform both of these groups with respect to

\section{fghi}

and obtain two new groups, as $a g . b h . c i . d f . e j$ is not found in either of the preceding groups, since the only substitutions in these groups beginning with $a g$. are

$$
\begin{aligned}
& a g \cdot b i \cdot c j \cdot d h \cdot e f \\
& a g \cdot b f \cdot c h \cdot d j \cdot e i
\end{aligned}
$$


and $a g . b j . c f . d i . e h$ is not found in any one of the three preceding groups, since all the substitutions in these groups which begin with $a g$. are

$$
\begin{aligned}
& a g \cdot b i \cdot c j \cdot d h \cdot e f \\
& a g \cdot b f \cdot c h \cdot d j \cdot e i \\
& a g \cdot b h \cdot c i \cdot d f \cdot e j
\end{aligned}
$$

Since $g j . l i i$ and $f g h i$ are contained in $(a b c d e)_{20}(f g h i j)_{20}$ they transform the given base into itself, we have therefore exhausted the possible one hundred substitutions and found only one distinct group. Our theorem is thus proved.

An independent proof of these theorems may be outlined as follows. The non-primitive groups in question must contain $(a b c d e)$ cyc ( $f g h i j)$ cyc, as a common self-conjugate subgroup. They must therefore be subgroups of a group whose order is $20 \cdot 20 \cdot 2=800$ and this group must have a 25-1 correspondence to some non-primitive group in eight letters, since a different letter corresponds to each substitution of the form abcde. The required group is easily found to be

$$
(a e . b f \cdot c g . d h)(a b c d) \text { cyc }(e f g h) \text { cyc }
$$

Since this group has the following non-primitive subgroups

$\begin{array}{cc}\text { Order. } & \text { Number of subgroups. } \\ 32 & 1 \\ 16 & 2 \\ 8 & \mathbf{5} \\ 4 & 2 \\ \mathbf{2} & \mathbf{1}\end{array}$

- which are not conjugate with respect to substitutions in it, there must be the same numbers of distinct non-primitive subgroups of the required type in the given group of order 800 , and the orders of these are found by multiplying the orders of the given subgroups by 25 . The numbers of the subgroups of the last three orders constitute the proofs of our theorems.

It may be remarked that the list to which we referred in the first paragraph contains all the groups which we have just found, but that it contains, in addition to these, six which are not distinct from others in the list. $200_{6}$ and $200_{7}$ are identical, but in all other cases the groups which should be deleted are conjugate to others already in the list.

Michigan University, October, 1894. 